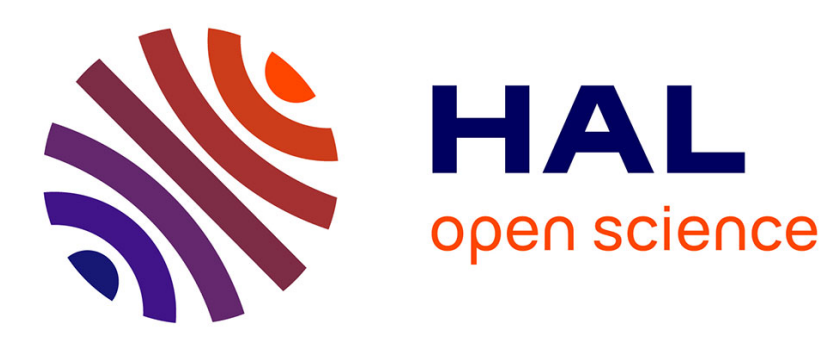

\title{
Thermodynamic model of the coupled valence and spin state transition in cobaltates
}

Yohann Breard, Vincent Hardy, François Guillou

\section{To cite this version:}

Yohann Breard, Vincent Hardy, François Guillou. Thermodynamic model of the coupled valence and spin state transition in cobaltates. Journal of Physics: Condensed Matter, 2020, 33, 095801 (8pp). 10.1088/1361-648X/abcbd9 . hal-03022161

\section{HAL Id: hal-03022161 \\ https://hal.science/hal-03022161}

Submitted on 24 Nov 2020

HAL is a multi-disciplinary open access archive for the deposit and dissemination of scientific research documents, whether they are published or not. The documents may come from teaching and research institutions in France or abroad, or from public or private research centers.
L'archive ouverte pluridisciplinaire HAL, est destinée au dépôt et à la diffusion de documents scientifiques de niveau recherche, publiés ou non, émanant des établissements d'enseignement et de recherche français ou étrangers, des laboratoires publics ou privés. 


\title{
Thermodynamic model of the coupled valence and spin state transition in cobaltates
}

\author{
V. Hardy, Y. Bréard, \\ Normandie Univ, ENSICAEN, UNICAEN, CNRS, CRISMAT, 14000 Caen, France \\ F. Guillou. \\ Inner Mongolia Key Laboratory for Physics and Chemistry of Functional Materials, College of Physics and \\ Electronic Information, Inner Mongolia Normal University, 81 Zhaowuda Road, Hohhot, 010022, Inner \\ Mongolia, China
}

\begin{abstract}
A class of cobalt-based oxides exhibits a peculiar type of transition, entangling valence and spin state degrees of freedom of $4 \mathrm{f}$ and $3 \mathrm{~d}$ elements. It constitutes one of the most spectacular illustrations of the interplay between charge, spin and lattice degrees of freedom in strongly correlated materials. In this work, we present a thermodynamic model capable to reproduce the main features of this transition. Our approach is based on the minimization of a free energy combining the contributions of two sublattices and the interaction between them. The coupling energies introduced in the model are related to well-known chemical pressure effects in the perovskite structure. The results of this model are compared to experimental data derived from x-ray absorption spectroscopy.
\end{abstract}

\section{Introduction}

A quite unique type of transition takes place in a class of mixed valent cobalt oxides, involving both a change in the mean valence of cobalt $\left(\mathrm{Co}^{3+} / \mathrm{Co}^{4+}\right)$ and in the spin state of $\mathrm{Co}^{3+}$ (low-spin / high-spin) (for an overview, see for instance [1,2] and references therein). This « valence and spin state transition » (VSST) strongly impacts all the physical properties, e.g., the magnetic susceptibility, the heat capacity, as well as the electrical and thermal conductivities [3-12]. There is no change of space group associated to this transition, but one observes a large reduction in the unit cell volume when crossing the VSST upon cooling [3,10,13-15]. Prominent signatures of such an isomorphic transition are observed in all the lattice-related physical quantities, such as the coefficient of thermal dilation, the sound velocity and so on [16]. Moreover, it must be emphasized that this transition can be observed not only by varying the temperature but also the magnetic field and the pressure [13,17-18]. One of the hallmarks of the VSST is that it can be very abrupt around the temperature of transition $\left(T_{t r}\right)$, leading to step-like changes in magnetization, entropy and unit cell volume, which make it entering the class of first-order transitions (FOT) [3]. 
The concomitance between the two facets of the transition (i.e., the valence and spin state aspects) has been widely documented, in particular by x-ray absorption spectroscopy (XAS) [1,9,19-23] and magnetic circular dichroism (XMCD) [2], and it is now a wellestablished fact. Basically, it is obvious that these phenomena are not just concomitant and there must be a coupling between them. At the present stage, however, the fact is that the nature of this coupling and the mechanisms driving the development of the VSST remain obscure. One can get clues about the underlying mechanisms by inspecting the structural and chemical features of the compounds showing a VSST. After the first observation of this type of transition in $\operatorname{Pr}_{0.5} \mathrm{Ca}_{0.5} \mathrm{CoO}_{3}$, [3] it was reported to take place within a wider range of compositions, namely $\left(\operatorname{Pr}_{1-y} \mathrm{R}_{\mathrm{y}}\right)_{1-\mathrm{x}} \mathrm{Ca}_{\mathrm{x}} \mathrm{CoO}_{3}$ with $\mathrm{R}=\mathrm{Sm}, \mathrm{Eu}, \mathrm{Gd}, \mathrm{Tb}, \mathrm{Y}$, and $0.2 \leq \mathrm{x} \leq 0.5$ as well as $0.0 \leq \mathrm{y} \leq 0.3[5,7,13,24-25]$. Importantly, all these compounds have a perovskite structure with both Pr and Ca on the A sites [26].

A decisive step in the investigations of the VSST came in 2010, with strong indications pointing towards the presence of tetravalent $\operatorname{Pr}$ cations at temperatures lower than $T$ tr, supported by DFT calculations [27] and several experimental techniques : heat capacity [8], neutron powder diffraction [15] and spectroscopy [19] data all converged towards this conclusion. Nowadays, there is a general consensus about the fact that a VSST transition requires, at low temperature $\left(T<T_{t r}\right)$, the stabilization of a mixed valence $\operatorname{Pr}^{3+} / \operatorname{Pr}^{4+}$ as well as $\mathrm{Co}^{3+}$ being in a low-spin (LS) state. Increasing the temperature destabilizes such a ground state by favoring (i) intra-atomic electronic transition within the $\mathrm{Co}^{3+}$ (yielding intermediate or high spin state, referred to as IS and HS, respectively), and (ii) electronic reorganization within the valence shells to make Pr recovering its usual trivalent state. Experimentally, one observes that these transformations often concentrates at the transition temperature $T_{t r}$. To understand how the VSST can be so sharp, one needs to address in more detail the link between the « valence » and « spin state » aspects of the transition.

\section{Position of the problem}

So far, only two kinds of modeling approaches have been explored for the VSST. Quite recently, Kuneš et al. [28-29] have developed a vision in terms of exciton condensation: the $\mathrm{Co}^{3+}$ spin-state part of the transition is treated as an excitonic process, while the Pr ions play the role of a charge reservoir with respect to the Co bands. It was reported that LDA+U calculations can account for the existence of a transition combining valence and spin-state 
degrees of freedom. In particular, sharp transitions mimicking the experimental ones can be reproduced as a function of the applied magnetic field [29]. However, a quantitative comparison to experimental data has not been attempted so far, to the best of our knowledge.

An alternative thermodynamical approach was used a few years earlier by Maryško et al. and Ikeda et al. [30-31]. It was inspired from the work developed by Biernacki et al. for $3 \mathrm{~d}^{6}$-based materials [32]. Basically, it consists in introducing an energy term reflecting the structural distortion associated to the spin state transition, within the usual treatment of a twolevels systems (namely LS and IS in the original study). This term leads to a progressive shrinking of the LS-IS gap as the transition develops, which makes it to accelerate. However this approach suffers from two major flaws. First, there is no coupling with the Pr and the valence degree of freedom. Second, an acceleration of the spin state transition is at odds with the widely documented case of the perovskite cobaltates, for which the transition rather tends to slow down along its development [33-34]. Indeed, there are many studies pointing that the LS-IS or LS-HS gap increases as the spin state transition spreads upon warming [35-37].

The aim of the present paper is to build a statistical model able to reproduce the valence and spin state facets of the VSST as a function of the temperature. To do so, we will adapt models previously used to address independently either the valence or the spin state transitions (referred to as the VT and SST, respectively). For the spin state transition, various approaches have been explored, including excitonic models [38], Ising-like models [39], and the theory of regular solutions [40], to only cite a few. Actually, most of these models turn out to yield very similar results, as discussed by Zimmermann et al. [41]. We will adopt the statistical approach of the regular solutions that was shown to be particularly versatile; it can indeed account for either the sharp transitions often seen in $\mathrm{Fe}^{2+}$-based molecular compounds like [Fe(phen)2 (NCS)2] [41], or the gradual transitions found in some $\mathrm{Co}^{3+}$-based oxides like $\mathrm{LaCoO}_{3}$ [34]. To address the valence part of the transition, we will consider the interconfigurational fluctuation (ICF) model [42-44]. It is the most widely used approach to

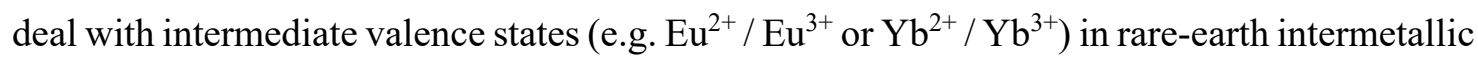
compounds [42-47].

The VSST corresponds to a tricky situation involving two subsystems (i.e., the Pr subsystem where the valence is varied, and the $\mathrm{Co}^{3+}$ sub-system for which the variable is the spin state). Moreover, these two sub-systems are coupled to each other. To address this problem, our approach is to introduce an overall free energy including the contributions of each sublattice as well as their interaction. We consider there is no cause or consequence between 
the valence and spin state parts of the VSST ; their coupled evolutions should just result from the minimization of the total free energy.

Our objective was to describe the VSST via the evolution with temperature of the average valence of $\mathrm{Pr}$ and of the average spin state of $\mathrm{Co}^{3+}$. The relevancy of the model was assessed by comparing its predictions to experimental data derived from XAS, which is the most suitable technique to characterize quantitatively the VSST. For this purpose, we selected the system $\left(\mathrm{Pr}_{1-\mathrm{y}} \mathrm{Sm}_{\mathrm{y}}\right)_{1-\mathrm{x}} \mathrm{Ca}_{\mathrm{x}} \mathrm{CoO}_{3}$ [48], which is one of the most widely studied in the VSST literature $[2,5,7,9,11,49]$.

\section{Qualitative interpretation}

First of all, let us tackle a qualitative description of the mechanism at the origin of the VSST. In what follows, we use two assumptions : (i) the intermediate valence of Pr is regarded as a simple mixture $\operatorname{Pr}^{3+} / \operatorname{Pr}^{4+}$ (even though the exact nature of $\operatorname{Pr}^{4+}$ might be more subtle, involving some degree of hybridization with the $\mathrm{O} 2 p$ valence band) $[13,20,50]$; (ii) the excited spin state of $\mathrm{Co}^{3+}$ is associated to a mixture LS/HS, rather than a IS state. After more than fifty years of intense controversy in $\mathrm{LaCoO}_{3}$, one can indeed state that this LS/HS description has become the most reliable $[2,9,36,37,51,52]$.

We propose a qualitative interpretation of the VSST that is based on chemical pressure effects typical of the perovskite structure. In a perovskite structure $\mathrm{ABO}_{3}$, where $\mathrm{B}$ denotes the octahedral sites (e.g., $\mathrm{B}=\mathrm{Co}$ in our case, while $\mathrm{A}=\mathrm{Pr}, \mathrm{Sm}$ and $\mathrm{Ca}$ ), effects of chemical pressure can be addressed in a first approximation by considering the average ionic radii at the A and B sites, denoted as $\left\langle r_{A}\right\rangle$ and $\left\langle r_{B}\right\rangle$, respectively. The radii of all involved cationic species are shown in Figure 1. The main features are: (i) a decrease in radius as the valence is increased or the spin state is decreased (for a given element); (ii) values of $r_{A}$ that are virtually equal for $\mathrm{Pr}^{3+}$ and $\mathrm{Ca}^{2+}$, whereas it is smaller for $\mathrm{Sm}^{3+}$.

Two main effects of chemical pressure (referred to as mechanisms afterwards) must be considered: (1) the presence of large $\left\langle r_{A}\right\rangle$ favors the stabilization of large $\left\langle r_{B}\right\rangle$, and vice versa ; (2) for a given $\left\langle r_{A}\right\rangle$, the appearance of a large $r_{B}$ at some B sites favors the existence of small $r_{B}$ at the neighboring sites (and vice versa). Mechanism 1 is mainly a matter of stability of the structure, that is more usually taken into consideration via the tolerance factor [53]. This mechanism is very well-known in the case of cobaltates like $\left(\mathrm{La}_{1-\mathrm{x}} \mathrm{Sr}_{\mathrm{x}}\right) \mathrm{CoO}_{3}$ or $\mathrm{RCoO}_{3}$ for which changing $\left\langle r_{A}>\right.$ allows to favor either LS or HS states for the $\mathrm{Co}^{3+}$ on the $\mathrm{B}$ 
sites [54-56]. Mechanism 2 relies on the fact that all the B sites are intimately connected to each other (via the oxygen framework) owing to the substantial covalent character of the B-O bonds (whereas the $\mathrm{A}-\mathrm{O}$ ones are essentially ionic). A large $r_{B}$ at a site displaces oxygens towards surrounding B sites, which thus become less favorable to host another large $r_{B}$. This process is particularly relevant to the development of the spin state transition (SST) in cobaltates: the emergence of HS at a site displaces oxygens towards $\mathrm{Co}^{3+}$ in neighboring sites ; this squeezing reinforces the stability of LS in the adjacent sites and, thereby, it hinders the development of the SST $[5,33,34,52,57]$. Beyond the size of $r_{B}$ as such, the phenomenon can be reinforced by the stronger covalent nature of the $\mathrm{Co}^{3+}(\mathrm{LS})-\mathrm{O}^{2-}$ bond compared to that of $\mathrm{Co}^{3+}(\mathrm{HS})-\mathrm{O}^{2-}[34,57,58]$.

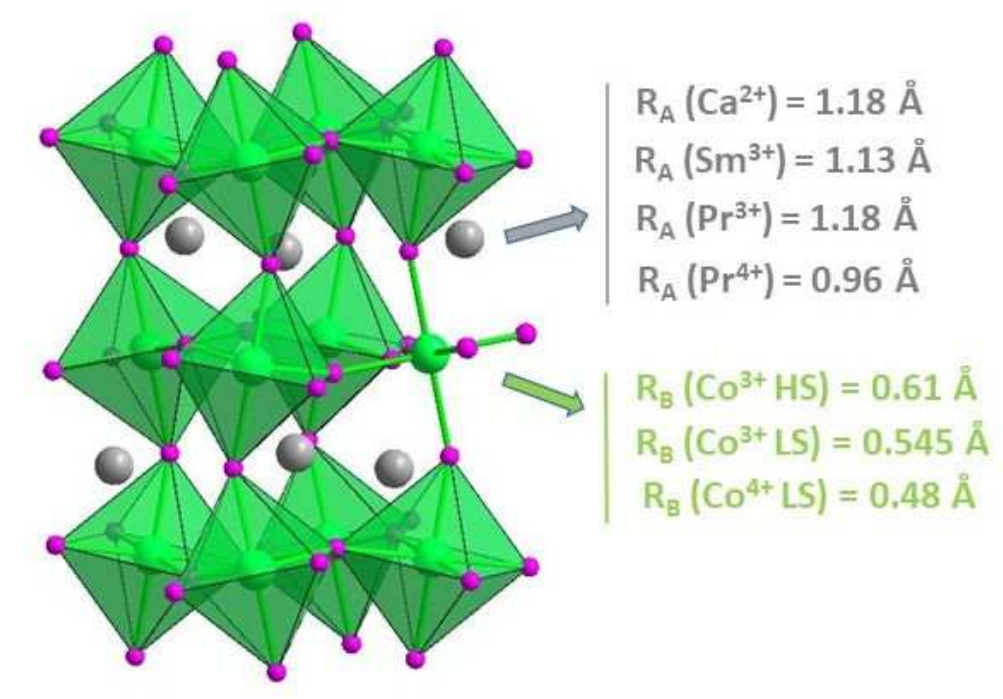

Figure 1 : Atomic structure of the orthorhombic perovskite $\mathrm{ABO}_{3}$ ( $\mathrm{SG}$ : Pnma) typical of the $\left(\operatorname{Pr}_{1-}\right.$ ySmy $)_{1-x} \mathrm{Ca}_{\mathrm{x}} \mathrm{CoO}_{3}$ compounds. The shown tilting of the $\mathrm{BO}_{6}$ octahedra corresponds to $y=0.3$. The cations at the $\mathrm{A}$ and $\mathrm{B}$ sites are shown by grey and green circles, respectively. The oxygen atoms are the small purple circles. The right panel gives the ionic radii of the cations in ninefold and sixfold coordination, for the A and B sites, respectively.

On the basis of these two mechanisms, we suggest the following scenario: Being driven by the usual SST process, the appearance of the first $\mathrm{Co}^{3+} \mathrm{HS}$ below $T_{t r}$-having a $r_{B}$ larger than that of $\mathrm{Co}^{3+}$ LS- tends to favors large $r_{A}$ (via mechanism 1), thus in particular the conversion of $\mathrm{Pr}^{4+}$ to $\mathrm{Pr}^{3+}$. Still via mechanism 1, this can trigger a feedback phenomenon (large $\mathrm{Pr}^{3+}$ favoring large $\mathrm{Co}^{3+} \mathrm{HS}$ ) which pushes further the development of the LS $\rightarrow \mathrm{HS}$ transition in $\mathrm{Co}^{3+}$. Such a synergy could be at the origin of the rapid changes in the populations of $\mathrm{Co}^{3+} \mathrm{LS}, \mathrm{Co}^{3+} \mathrm{HS}, \mathrm{Pr}^{3+}, \mathrm{Pr}^{4+}$ and $\mathrm{Co}^{4+}$ (imposed by charge balance) that take place at $T_{t r}$. In 
principle, one should also take into account that the appearance of $\mathrm{Co}^{3+} \mathrm{HS}$ can act in an opposite way by disfavoring new LS $\rightarrow$ HS conversion because of mechanism 2 . However, this latter effect should be limited around $T_{t r}$, since it is counterbalanced by the concomitant appearance of $\mathrm{Co}^{4+}$ which possess a $r_{B}$ smaller than that of $\mathrm{Co}^{3+}$. After the completion of the jump at $T_{t r}$, all $\mathrm{Pr}$ are transformed to $\mathrm{Pr}^{3+}$, so no more synergy is expected between the sublattices A and B ; The evolution of the SST upon further warming turns back to a regime where it is mainly driven by the competition between the large $r_{B}$ of $\mathrm{Co}^{3+} \mathrm{HS}$ and the smaller one of $\mathrm{Co}^{3+}$ LS via mechanism 2; This is expected to slow down the SST in a way similar to that found in $\mathrm{LaCoO}_{3}$.

\section{Thermodynamic approach}

The VSST involves changes in the amount of five species $\left(\mathrm{Co}^{3+} \mathrm{LS}, \mathrm{Co}^{3+} \mathrm{HS}, \mathrm{Co}^{4+}\right.$, $\operatorname{Pr}^{3+}$ and $\operatorname{Pr}^{4+}$ ) but there are actually only two independent variables, that can be chosen to be $\left[\mathrm{Pr}^{3+}\right]$ and $\left[\mathrm{Co}^{3+} \mathrm{HS}\right][59]$. We propose a statistical model with two sublattices in interaction. The first one consists of the Pr on the A sites, having each a probability $m$ to be $\mathrm{Pr}^{3+}$ and (1$m)$ to be $\operatorname{Pr}^{4+}$. The second sublattice is made of the $\mathrm{Co}^{3+}$ on the $\mathrm{B}$ sites, having each a probability $n$ to be HS and (1-n) to be LS.

The free energy (normalized to one particle) for a set of particles which can be in states 1 or 2 (with a probability $x$ to be in state 1 ) can be written as:

$$
f(x)=x f_{1}+(1-x) f_{2}+u_{i n t}(x)-T s_{m i x}(x),
$$

where $f_{i}(i=1,2)$ is the free energy of one particle being in the state $i$. All terms in Eq. (1) are temperature dependent, including $x$ itself. This expression is derived from the theory of regular solutions [40], and it was shown to be consistent with several other approaches [41]. The quantities $u_{\text {int }}$ and smix correspond to the interaction energy and the entropy of mixing between particles, respectively. The general expression of the entropy term is $s_{\text {mix }}(x)=$ $-x \ln (x)-(1-x) \ln (1-x)$. The free energies can be evaluated from $f_{i}=-T \ln Z_{i}$, where $Z_{i}$ is the individual partition function associated to the state $i$. This partition function is derived from the diagram of energy levels (index $k$ ) of the particle in state $i: Z_{i}=\sum_{k} g_{i}^{k} e^{-\left(\varepsilon_{i}^{k}+\Delta\right) / T}$. In this expression, $g_{i}^{k}$ and $\varepsilon_{i}^{k}$ refer to the degeneracy and energy of the level $k$ of state $i$, respectively. By convention, $\varepsilon_{i}^{k=0}=0$ for the lowest level, and the energy $\Delta$ is 0 for the state 
( 1 or 2 ) which is the lowest in energy, while it is equal to the spacing between the lowest levels $(k=0)$ of the two states for the other ( 2 or 1$)$.

Let us now apply the general expression of Eq. (1) to each sublattice. For the B sublattice, we consider $x=n$, state $1=\mathrm{Co}^{3+} \mathrm{HS}$ and state $2=\mathrm{Co}^{3+}$ LS. The lowest state is $\mathrm{Co}^{3+}$ LS which defines the zero in energy (see Fig. 2). For state $1, f_{1}$ is derived from the energy diagram of $\mathrm{Co}^{3+} \mathrm{HS}$ in an octahedral $\left(\mathrm{O}_{\mathrm{h}}\right)$ environment $\left(t_{2 g}^{4} e_{g}^{2}\right)$. The combination of the crystalline electric field (CEF) and spin-orbit coupling (SOC) is expected to split the 15 sublevels mainly into a triplet, a quintet and a sextet [60]. These energy levels of HS are shifted by an energy gap $\Delta$ from LS (see Fig. 2 ). The energy $f_{1}$ is calculated from the expression $Z_{1}=Z\left(\mathrm{Co}^{3+} \mathrm{HS}\right)=\sum_{k} g_{1}^{k} e^{-\left(\varepsilon_{1}^{k}+\Delta\right) / T}$, using the energy levels $\varepsilon_{1}^{k}$ derived in [60]. As for state $2, \mathrm{Co}^{3+} \mathrm{LS}$ in $\mathrm{Oh}\left(t_{2 g}^{6}\right)$ is a singlet at the groundstate, leading to $f_{2}=0$. To account for the interaction among the $\mathrm{Co}^{3+}$ (LS or HS), we use the general form $u_{i n t}^{B}(n)=-J_{B} n^{2}$ $[38,41]$. The case $J_{B}>0$ corresponds to a "positive » cooperative effect, which tends to accelerate the transition (since $n$ is associated to the upper state); This is the case of many $\mathrm{Fe}^{2+}$ based molecular compounds [41]. In the perovskite cobaltates like $\mathrm{LaCoO}_{3}$, or those showing VSST, one rather expects $J_{B}<0$; This is a «negative » cooperative effect (associated to the mechanism 2 described in our qualitative discussion) which impedes the development of the transition [34]. In the end, for the B sublattice, one arrives at the following expression for the individual free energy :

$$
f_{B}=n\left[-T \ln Z\left(\mathrm{Co}^{3+} \mathrm{HS}\right)\right]-J_{B} n^{2}+T[n \ln (n)+(1-n) \ln (1-n)]
$$

We emphasize that this expression is equivalent to those reported in the early papers on the SST [41]. It also deserves to be emphasized that the quadratic term $\left(-J_{B} n^{2}\right)$ in Eq. (2) is at the origin of the effective gap $\left(\Delta-2 J_{B} n\right)$ often used to describe the LS and HS populations in $\mathrm{Fe}^{2+}$-based materials via a modified Boltzmann law [61].

Let us now turn to the free energy of the A sublattice. We consider in this case $x=m$, state $1=\operatorname{Pr}^{3+}$ and state $2=\operatorname{Pr}^{4+}$. Note that the groundstate of $\operatorname{Pr}^{4+}$ is assumed to lie above that of $\operatorname{Pr}^{3+}$ (see Fig. 2). In the cubo-octahedral environment of the A site, $\operatorname{Pr}^{3+}\left(4 \mathrm{f}^{2}, J=4\right)$ undergoes a splitting in 9 singlets, while $\operatorname{Pr}^{4+}\left(4 \mathrm{f}^{1}, J=5 / 2\right)$ exhibits 3 doublets. We used the energy levels given in ref [62] to evaluate $Z\left(\operatorname{Pr}^{3+}\right)$ and $Z\left(\operatorname{Pr}^{4+}\right)$ [63]. The ICF model introduces an effective temperature $T^{*}=\sqrt{T^{2}+T_{f}^{2}}$, where $T_{f}$ is the valence fluctuation temperature [43]. This phenomelogical parameter is assumed to reflect a broadening of the energy levels 
that can account for the stabilization of a mixed valence. The temperature $T^{*}$ replaces $T$ in the evaluation of thermodynamical quantities, in particular for the partition functions $Z_{i}=$ $\sum_{k} g_{i}^{k} e^{-\left(\varepsilon_{i}^{k}+\Delta\right) / T^{*}}$. As shown in Fig. 2, one considers $\Delta=0$ for $\operatorname{Pr}^{3+}$ and $\Delta=\delta$ for $\operatorname{Pr}^{4+}$. Moreover, an interaction term similar to that previously introduced for the B sublattice can be added. Keeping in mind that $(1-m)$ corresponds to the upper state, this interaction term takes the form $u_{i n t}^{A}(m)=-J_{A}(1-m)^{2}$. This leads to a generalized expression for the $\mathrm{A}$ sublattice :
$f_{A}=m\left[-T^{*} \ln Z\left(\operatorname{Pr}^{3+}\right)\right]+(1-m)\left[-T^{*} \ln Z\left(\operatorname{Pr}^{4+}\right)\right]-J_{A}(1-m)^{2}+T^{*}[m \ln (m)+(1-$
$m) \ln (1-m)]$

Importantly, this expression is consistent with those used in the papers on $\mathrm{Eu}$ - or $\mathrm{Yb}$ based compounds $[44,46,47,64]$, where the population of the two valence states are given by a Boltzmann law involving a gap of the form $E_{\text {ex }}=\delta-2 J_{A}(1-m)$ [65]. In the case of the VSST materials, however, there is no obvious justification for the occurrence of such a coupling among the A sublattice. The cations in the A sublatice are less in interaction that those of the B sublattice, which have a higher degree of covalency. Thereby, in order to restrict as much as possible the number of parameters in the model, we will consider thereafter $J_{A}=$ 0 , leading to

$f_{A}=m\left[-T^{*} \ln Z\left(\operatorname{Pr}^{3+}\right)\right]+(1-m)\left[-T^{*} \ln Z\left(\operatorname{Pr}^{4+}\right)\right]+T^{*}[m \ln (m)+(1-m) \ln (1-m)]$

Let us now turn to the interaction energy between the two sublattices. It basically derives from mechanism 1, for which the large $\mathrm{Pr}^{3+}$ on the A sites favors the emergence of large $\mathrm{Co}^{3+} \mathrm{HS}$ on the B sites, and vice versa. In a way similar to that used within each sublattice, this interaction can be accounted for by a term of the form

$$
f_{A B}=-J_{A B} n m
$$

For $J_{A B}>0$, this term reflects a positive synergy between the VT and SST transformations. 

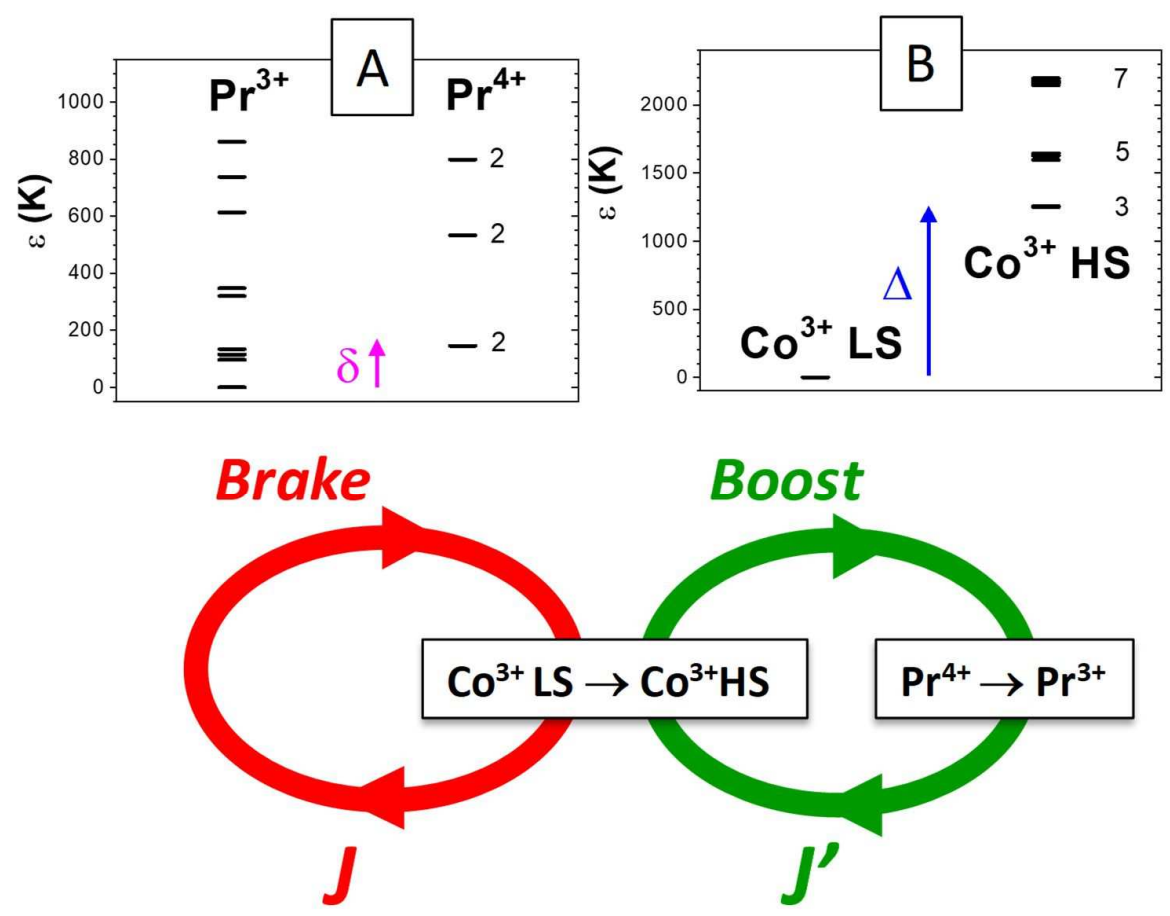

Figure 2 : Energy diagrams of $\mathrm{Pr}^{3+}$ and $\mathrm{Pr}^{4+}$ at the A sites of the perovskite structure, and of $\mathrm{Co}^{3+}$ (being either LS our HS) at the B sites. The levels for the Pr cations are those calculated in Ref. [62], while those of $\mathrm{Co}^{3+} \mathrm{HS}$ are taken from Ref. [60]. The lower panel is a schematic picture highlighting the competing mechanisms acting on the development of the VSST: a braking effect affecting the spin state transition of the $\mathrm{Co}^{3+}$ (controlled by the energy $J$ in the model), and a boosting effect originating from the synergy between the spin state (B sites) and valence state (A sites) aspects of the VSST (controlled by the energy $J$ ' in the model).

At this stage, one must combine the individual energies $(f)$ to get quantities representative of the cationic stoichiometry of a formula unit $(F)$. The important point is the balance between $F_{A}$ (proportional to the amount of $\operatorname{Pr}$ ) and $F_{B}$ (proportional to the amount of $\left.\mathrm{Co}^{3+}\right)$, that led us to introduce the parameter $\alpha=[\mathrm{Pr}] /\left[\mathrm{Co}^{3+}\right]$. Moreover, in order to deal only with positive values of coupling, we will consider $J=-J_{B}$ and $J^{\prime}=J_{A B}$. As a result, the overall free energy to be considered when addressing the VSST is :

$$
\begin{aligned}
& F(n, m)=n\left[-T \ln Z\left(\mathrm{Co}^{3+} \mathrm{HS}\right)\right]+T[n \ln (n)+(1-n) \ln (1-n)]+J n^{2}+\alpha\left\{m\left[-T^{*} \ln Z\left(\mathrm{Pr}^{3+}\right)\right]+\right. \\
& \left.(1-m)\left[-T^{*} \ln Z\left(\operatorname{Pr}^{4+}\right)\right]+T^{*}[m \ln (m)+(1-m) \ln (1-m)]\right\}-J^{\prime} n m
\end{aligned}
$$

\section{Results and discussion}

$F$ is a function of two variables $(n, m)$ and it contains five parameters $\left(T_{f}, \delta, \Delta, J, J^{\prime}\right)$. At each temperature, the couple of variables $(n, m)$ must take the values yielding minimization 
of $F$. In case of a genuine minimum (in the mathematical sense), one can set the partial derivatives of $F(n, m)$ equal to zero :

$$
(\partial F / \partial m)_{n}=0 \Leftrightarrow m=\left\{1+\left[Z\left(\operatorname{Pr}^{4+}\right) / Z\left(\operatorname{Pr}^{3+}\right)\right] \exp \left[-\left(J^{\prime} n\right) /\left(\alpha T^{*}\right)\right]\right\}^{-1}
$$

and

$$
(\partial F / \partial n)_{m}=0 \Leftrightarrow m=\left\{-T \operatorname{lnZ}\left(\mathrm{Co}^{3+} \mathrm{HS}\right)+2 J n+\ln [n /(1-n)]\right\} / J^{\prime}
$$

However, it must be kept in mind that a minimum of $F$ can be reached without zeroing its derivative, in particular when the solution corresponds to one of the boundaries of the range of allowed values. In the present case, one can anticipate that this may occur for $n$ at low- $T$ (since the $\mathrm{Co}^{3+}$ are expected to be mainly LS below $T_{t r}$, i.e., $n \rightarrow 0$ ). For relevant values of parameters (see below) and experimental values of $(n, m)$, the fact is that $F(m)$ always exhibits one minimum within the range ]0,1[ at all temperatures, so Eq. (7.1) is legitimate. On the contrary, $F(n)$ is a monotonously increasing function at low- $T$, which can challenge the relevance of Eq. (7.2) in some regimes. Therefore, one is led to rather adopt a numerical approach to determine, at each temperature, the couple $(n, m)$ yielding the lowest value of $F$.

The procedure we followed is divided in several steps : (i) Choice of a set of parameters ( $\left.T_{f}, \delta, \Delta, J, J^{\prime}\right)$; (ii) At selected values of $T$, calculation of $m(n, T)$ from Eq. (7.1); (iii) Incorporation into Eq. (6) to get $F(n, T)$; (iv) Numerical derivation of the minimum of $F(n)$ at each $T$, which yields values of $n_{\min }(T)$; (v) Calculation of $m_{\min }(T)$ from $n_{\min }(T)$ by using the above $m(n, T)$ expression; (vi) Comparison of the obtained set $[n(T), m(T)]$ to the XAS data. This sequence is repeated by adjusting the input parameters $\left(T_{f}, \delta, \Delta, J, J^{\prime}\right)$ till reaching satisfying agreement with the data. 
Figure 3 : Total free energy as a function of $n\left(=\left[\mathrm{Co}^{3+} \mathrm{HS}\right] /\left[\mathrm{Co}^{3+}\right]\right)$, calculated at various temperatures. The parameters $\left(T_{f}, \delta, \Delta, J, J^{\prime}\right)$ are those leading to the best agreement with the XAS data (see text). The lower panel shows the difference $F(n)-F(n=0)$ to better visualize the shape of the curves at several temperatures. The position of the minimum of each curve is marked by a triangle.

In the literature, the few XAS studies addressing both the VT and SST aspects have reported very consistent features [2,9,19,20,22]. For the present study, we chose to consider the results obtained on $\left(\operatorname{Pr}_{0.7} \mathrm{Sm}_{0.3}\right)_{0.7} \mathrm{Ca}_{0.3} \mathrm{CoO}_{3}$ which possess closely spaced experimental points around the transition $\left(T_{t r}\right)[9]$. For the parameter $\alpha=[\operatorname{Pr}] /\left[\mathrm{Co}^{3+}\right]$, we evaluated its value at $T>T_{t r}$ when $\operatorname{Pr}$ is purely trivalent, in such a way that the chemical formula can be written as $\operatorname{Pr}_{0.49}^{3+} \mathrm{Sm}_{0.21}^{3+} \mathrm{Ca}_{0.30}^{2+} \mathrm{Co}_{0.7}^{3+} \mathrm{Co}_{0.3}^{4+} \mathrm{O}_{3}^{2-}$, leading to $\alpha=0.49 / 0.7=0.7$.

Fortunately, the five parameters to be adjusted $\left(T_{f}, \delta, \Delta, J, J^{\prime}\right)$ are not completely free parameters. First, the LS-HS gap $\Delta$ is intimately related to the average A site radius $\left\langle r_{A}\right\rangle$. From previous studies on various series, such as $\mathrm{La}_{1-x} \mathrm{Eu}_{x} \mathrm{CoO}_{3}[66]$ and $\mathrm{RCoO}_{3}(\mathrm{Ln}=\mathrm{Dy}, \mathrm{Gd}$, $\mathrm{Sm}, \mathrm{Nd}, \mathrm{Pr}$ and La) $[55,56]$, the $\Delta$ value in our case is expected to lie between 1000 and 2000 
$\mathrm{K}$. Second, there is a relationship between $T_{f}$ and $\delta$ which is fixed by the experimental value of $m(T=0) \sim 0.73$. Third, in order to be in accordance with the steps present on both $m(T)$ and $n(T)$ at the transition, the $m(n)$ expressions calculated from Eqs. (7.1) and (7.2) at $T=T_{t r}$ must be tangent to each other. One can even state that the overlapping segment of $m(n)$ curves must be consistent with the experimental values of $m$ and $n$ along the jumps [65]. This induces a relationship between the three parameters $\left(\Delta, J, J^{\prime}\right)$. All these constraints help us to reduce the domain of parameters to be explored.

By adopting a trial-and-error process, we found that the following set of parameters is able to reproduce properly the main features of the VSST : $T_{f}=200 \mathrm{~K} ; \delta=145 \mathrm{~K} ; \Delta=1250$ $\mathrm{K} ; J=100 \mathrm{~K} ; J^{\prime}=1165 \mathrm{~K}$. Figure 3 shows plots of $F(n)$ at selected $T$ values for this set of parameters. At $T<T_{t r}$, one observes a monotonically increasing function starting from $n=0$. Approching $T_{t r}$, a minimum in $F(n)$ takes place at $n \neq 0$. Around $T_{t r}$, a second minimum emerges, and one observes a rapid swap between them as $T$ is varied. It turns out that the positions of these minima correspond to the lower and upper boundaries of the jump in $n(T)$ at the transition. At $T_{t r}$, the $F(n)$ function exhibits a very shallow minimum, which is the behavior expected for a FOT. Then, for $T>T_{t r}, F(n)$ shows a single minimum whose position increases with temperature. For all these $n_{\min }(T)$, we calculated the associated $m_{\min }(T)$ values via Eq. (7.1).

Figure 4 shows the results of this analysis, compared to the XAS data. One can underline a reasonable, overall consistency between the model and the data. From $T=5 \mathrm{~K}$ till $T_{t r}$, there is a very weak change in $m$ and $n$ (just a rounding on the low- $T$ wing of the transition). At $T_{t r}$, one gets concomitant jumps in the spin state of $\mathrm{Co}^{3+}$ and the valence of $\mathrm{Pr}$; the one on « $m$ » is complete (i.e., reaching $m=1$ ) whereas the jump on « $n »$ is only partial. Above $T_{t r}$, $n$ continues to increase up to room temperature, but much more progressively. 
Figure 4 : Temperature dependence of the fractions of $\mathrm{HS}$ in $\mathrm{Co}^{3+}$ and of trivalent $\mathrm{Pr}$. The symbols are XAS data, while the line is derived from the model for a certain set of parameters (see text).

We claim that the first-order character of the VSST corresponds to a transient acceleration originating from a synergy between two coupled degrees of freedom : the valence state (of $\mathrm{Pr}$ ) and the spin state ( $\mathrm{of} \mathrm{Co}^{3+}$ ). Actually, $\mathrm{Pr}^{4+}$ can be regarded as the fuel of a booster process stimulating the transformation $\mathrm{Co}^{3+} \mathrm{LS} \rightarrow \mathrm{Co}^{3+} \mathrm{HS}$; such a process is expected to end when all the $\operatorname{Pr}^{4+}$ present in the low- $T$ regime are consumed, which is well compatible with the much smoother increase of $\mathrm{Co}^{3+} \mathrm{HS}$ versus temperature above the VSST.

Our approach in terms of minimization of the free energy $F$ is made relevant by the fact that the VSST exhibits virtually no hysteresis [65]. It is worth specifying that the VSST is not associated to any magnetic ordering nor any change of space group. It is the synergy between two coupled degrees of freedom that can yield a so sharp transition, conferring to the VSST a first-order character. In this regard, it must also be underlined that this sharpness is not an intrinsic feature of the VSST, since this type of transition can become broad for some compositions $[1,5,12]$.

Let us now comment on the obtained parameters $\left(T_{f}, \delta, \Delta, J, J^{\prime}\right)$, even though it must be clear that this set of values cannot be regarded as the unique, optimal solution : this is due, first, to the used procedure (trial-and-error) and, second, to a sizeable interdependence between 
certain parameters. It remains that comparison to the literature is possible for some of these parameters. First, the $\Delta$ value of $1250 \mathrm{~K}$ for a $\left\langle r_{A}\right\rangle=1.17 \AA$ is well in line with the values reported in previous studies of cobaltates [65]. Second, the interaction $J$ can be compared to previous works on $\mathrm{LaCoO}_{3}$, even though different types of analysis were used. In 2005, Kyomen [67] described the anticooperative effect along the SST by introducing an effective gap of the form $\Delta_{e f f}=\Delta+\sum_{i=1}^{3} \Delta_{i} n^{i}$. In first approximation, our $J$ value $(100 \mathrm{~K})$ can be compared to the linear term of the polynomial development $\left(\Delta_{1}=305 \mathrm{~K}\right)$, and one observes they are indeed of the same order of magnitude. However, in $\mathrm{LaCoO}_{3}$, the other $\Delta_{\mathrm{i}}$ also get significant positive values (e.g., $\Delta_{2}=556 \mathrm{~K}$ ) in such a way that $\Delta_{\text {eff }}$ diverges before reaching $n \sim 0.5$, contrary to the case of the present study. It is reasonable to consider that the repulsive interaction between the $\mathrm{Co}^{3+} \mathrm{HS}$ is probably weakened in the mixed-valent VSST compounds, owing to a dilution effect by the $\mathrm{Co}^{4+}$ (not present in $\mathrm{LaCoO}_{3}$ ). About the $\delta$ value (shift in energy between $\operatorname{Pr}^{3+}$ and $\operatorname{Pr}^{4+}$ ), the only available data in the literature deals with intermetallics, that are quite different materials. It is worth noting, however, that our $\delta \sim 145 \mathrm{~K}$ is of the same order of magnitude as the values reported for $\mathrm{Yb}^{2+} / \mathrm{Yb}^{3+}(\delta \sim 170-550 \mathrm{~K})[42,46]$ and $\mathrm{Eu}^{3+} /$ $\mathrm{Eu}^{2+}(\delta \sim 170-800 \mathrm{~K})[43,47]$ in intermetallic compounds. The same also holds for $T_{f}=200 \mathrm{~K}$ which is similar to values reported for the valence transition in $4 \mathrm{f}$ compounds, for instance 50$200 \mathrm{~K}$ for $\mathrm{Eu}^{3+} / \mathrm{Eu}^{2+}$ [47]. Finally, the only parameter for which there is absolutely no point of comparison in the literature is the effective interaction $J^{\prime}$ that we introduced between $\operatorname{Pr}^{3+}$ and $\mathrm{Co}^{3+} \mathrm{HS}$.

Apart from the uncertainty related to the adjustment procedure, the reliability of our parameter values is also impacted by various weaknesses in the model itself. First of all, one can see in Fig. 4 that the calculated $m$ value does not saturate to 1 as it is experimentally observed (it rather tends to slightly decrease as $T$ is increased up to $300 \mathrm{~K}$ ). This is inherent in our model where the gap between the groundstates of $\operatorname{Pr}^{3+}$ and $\operatorname{Pr}^{4+}$ has a fixed value. To account for the achievement of a purely $\operatorname{Pr}^{3+}$ state as the temperature is increased, one should enhance the effective gap $\delta$ along the development of the valence transition. This would correspond to the introduction of an interaction $J_{A}<0$ in Eq. (3) (as done in modified versions of the ICF model applied to Yb-based intermetallics [46,47], for instance). As discussed above, we have preferred to discard this term in a first approximation, for two main reasons : first, in order to better highlight the essence of the process by focusing on the key aspects, and, second, because we could not propose any clear justification for such an interaction term. It remains, however, that such a corrective term should improve a lot the agreement with the data. A 
second serious limitation of the model is that we did not take into account the variation in the cell volume at the transition. It turns out that the lattice expansion at $T_{t r}$ is sizeable, since it can reach $+1.5-2 \%$ upon warming $[3,10,13-15]$; The introduction of an elastic term in the free energy can help to better fit to the data, as shown in the case of molecular compounds, even though the impact remains moderate [41]. Two less serious drawbacks should also be noted : (i) the parameter $\alpha=[\operatorname{Pr}] /\left[\mathrm{Co}^{3+}\right]$ which is aimed to reflect the balance between the two sublattices should depend on $T$ since $\left[\mathrm{Co}^{3+}\right]=0.7+\left[\mathrm{Pr}^{4+}\right]$; (ii) in principle, a temperature dependence might also be invoked for all the other parameters, i.e. $\Delta(T), \delta(T)$, and $T_{f}(T)$. We think that these corrections should not affect the physical picture that is presented herein, but it is clear that they could improve the quality of the fitting.

\section{Conclusion}

We have proposed a phenomelogical model able to reproduce the main features of the VSST which takes place in a specific class of cobaltates. This model can be regarded as an extension of the statistical approaches already developed to deal with SST or VT separately. The energy terms we added derive from two well-known phenomena of chemical pressure in perovskites $\left(\mathrm{ABO}_{3}\right)$, which generate «steric-like» effects within and between the $\mathrm{A}$ and $\mathrm{B}$ sublattices.

Minimization of a general free energy $F$ involving the two sublattices and their interaction is found to reproduce the FOT-like character of the VSST. The basic factor at the origin of this behavior is a positive synergy between the VT (at the A sublattice) and the SST (at the B sublattice). At the present stage, our model remains rough and we suggest a few ways to improve such a thermodynamical description.

The VSST is a quite unique example of FOT without structural transition nor magnetic ordering. One interesting aspect of the developed approach is that it highlights why the VSST can only be observed in perovskite compounds containing both $\operatorname{Pr}$ and Co. However, the physical origin of the stabilization of a mixed valence $\operatorname{Pr}^{3+} / \operatorname{Pr}^{4+}$-which is at the heart of the VSST transition- is still unclear. In particular, the structural parameters yielding such an intermediate Pr valence in oxides are not yet well established. It is undeniable that these questions would deserve further specific attention. 


\section{References}

[1] J. Hejtmánek, Z. Jirák, O. Kaman, K. Knížek, E. Šantavá, K. Nitta, T. Naito, and H. Fujishiro, Eur. Phys. J. B. 86, 305 (2013).

[2] F. Guillou, K. Kummer, Y. Bréard, L. Hervé, and V. Hardy, Phys. Rev. B 95, 174445 (2017).

[3] S. Tsubouchi, T. Kyomen, M. Itoh, P. Ganguly, M. Oguni, Y. Shimojo, Y. Morii, and Y. Ishii, Phys. Rev. B 66, 052418 (2002).

[4] S. Tsubouchi, T. Kyomen, M. Itoh, and M. Oguni, Phys. Rev. B 69, 144406 (2004).

[5] T. Fujita, S. Kawabata, M. Sato, N. Kurita, M. Hedo, and Y. Uwatoko, J. Phys. Soc. Jpn 74, 2294 (2005).

[6] T. Naito, M. Sumomozawa, H. Sasaki, and H. Fujishiro, J. Phys. Conf. Ser. 150, 022062 (2009).

[7] T. Naito, H. Sasaki, and H. Fujishiro, J. Phys. Soc. Jpn 79, 034710 (2010).

[8] J. Hejtmánek, E. Šantavá, K. Knížek, M. Maryško, Z. Jirák, T. Naito, H. Sasaki, H. Fujishiro, Phys. Rev. B 82, 165107 (2010).

[9] F. Guillou, Q. Zhang, Z. Hu, C. Y. Kuo, Y.Y. Chin, H.J. Lin, C. T. Chen, A. Tanaka, L.H. Tjeng, and V. Hardy, Phys. Rev. B 87, 115114 (2013).

[10] K. Knížek, J. Hejtmánek, M. Maryško, P. Novák, E. Šantavá, Z. Jirák, T. Naito, H. Fujishiro, C. de la Cruz, Phys. Rev. B 88, 224412 (2013).

[11] V. Hardy, F. Guillou, and Y. Bréard, J. Phys.: Condens. Matter 25 (2013) 246003.

[12] D. Phelan, K. P. Bhatti, M. Taylor, S. Wang, and C. Leighton, Phys. Rev. B 89, 184427 (2014).

[13] T. Fujita, T. Miyashita, Y. Yasui, Y. Kobayashi, M. Sato, E. Nishibori, M. Sakata, Y. Shimojo, N. Igawa, Y. Ishii, K. Kakurai, T. Adachi, Y. Ohishi, and M. Takata, J. Phys. Soc. Jpn 73, 1987 (2004).

[14] P. Tong, Y. Wu, B. Kim, D. Kwon, J. M. S. Park, and B. G. Kim, Phys. Soc. Jpn 78, $034702(2009)$.

[15]. A. J. Barón-González, C. Frontera, J. L. García-Muñoz, J. Blasco, and C. Ritter, Phys. Rev. B 81, 054427 (2010).

[16] T. Naito, M. Sumomozawa, H. Sasaki, and H. Fujishiro, J. Phys. Conf. Ser. 200, 012137 (2010).

[17] T. Naito, H. Fujishiro, T. Nishizaki, N. Kobayashi, J. Hejtmánek, K. Knížek, Z. Jirák, J. Appl. Phys. 115, 233914 (2014).

[18] A. Ikeda, S. Lee, T. T. Terashima, Y. H. Matsuda, M. Tokunaga, and T. Naito, Phys. Rev. B 94, 115129 (2016).

[19] J. L. García-Muñoz, C. Frontera, A. J. Barón-González, S. Valencia, J. Blasco, R. Feyerherm, E. Dudzik, R. Abrudan, and F. Radu, Phys. Rev. B 84, 045104 (2011).

[20] J. Herrero-Martín, J. L. García-Muñoz, S. Valencia, C. Frontera, J. Blasco, A. J. BarónGonzález, G. Subías, R. Abrudan, F. Radu, E. Dudzik, and R. Feyerherm, Phys. Rev. B 84, 115131 (2011).

[21] H. Fujishiro, T. Naito, S. Ogawa, N. Yoshida, K. Nitta, J. Hejtmánek, K. Knížek, and Z. Jirák, J. Phys. Soc. Jpn 81, 064709 (2012).

[22] J. Herrero-Martín, J. L. García-Muñoz, K. Kvashnina, E. Gallo, G. Subias, J. A. Alonso, and A. J. Barón-González, Phys. Rev. B 86, 125106 (2012).

[23] J. M. Chen, J. M. Lee, S. C. Haw, S. A. Chen, V. Hardy, F. Guillou, S. W. Chen, C. Y. Kuo, C. W. Pao, J. F. Lee, N. Hiraoka, H. Ishii, K. D. Tsuei, and Z. Hu, Phys. Rev. B 90, 035107 (2014).

[24] H. Masuda, T. Fujita, T. Miyashita, M. Soda, Y. Yasui, Y. Kobayashi, and M. Sato, J. Phys. Soc. Jpn 72, 873 (2003). 
[25] A. V. Kalinov, O. Yu. Gorbenko, A. N. Taldenkov, J. Rohrkamp, O. Heyer, S. Jodlauk, N. A. Babushkina, L. M. Fisher, A. R. Kaul, A. A. Kamenev, T. G. Kuzmova, D. I. Khomskii, K. I. Kugel, and T. Lorenz, Phys. Rev. B 81, 134427 (2010).

[26] This coexistence $(\mathrm{Pr}, \mathrm{Ca})$ on the A sites allows to generate a mixed valence $\mathrm{Co}^{4+} / \mathrm{Co}^{3+}$ on the B sites (which will in turn accommodate the valence transition of Pr at the VSST), while keeping an average ionic radius on the A sites small enough to ensure a LS state for the $\mathrm{Co}^{3+}$ at low $\mathrm{T}$.

[27] K. Knížek, J. Hejtmánek, P. Novák, and Z. Jirák, Phys. Rev. B 81, 155113 (2010).

[28] J. Kuneš and P. Augustinský, Phys. Rev. B 90, 235112 (2014).

[29] A. Sotnikov and J. Kuneš, Phys. Rev. B 96, 245102 (2017).

[30] M. Maryško, Z. Jirák, K. Knížek, P. Novák, J. Hejtmánek, T. Naito, H. Sasaki, and H. Fujishiro, J. Appl. Phys. 109, 07E127 (2011).

[31] A. Ikeda, S. Lee, T. T. Terashima, Y. H. Matsuda, M. Tokunaga, and T. Naito, Phys. Rev. B 94, 115129 (2016).

[32] S. W. Biernacki and B. Clerjaud, Phys. Rev. B 72, 024406 (2005).

[33] M. A. Señaris-Rodríguez and J. B. Goodenough, J. Solid State Chem. 116, 224 (1995).

[34] T. Kyomen, Y. Asaka, and M. Itoh, Phys. Rev. B 67, 144424 (2003).

[35] R. A. Bari and J. Sivardière, Phys. Rev. B 5, 4466 (1972).

[36] M. W. Haverkort, Z. Hu, J. C. Cezar, T. Burnus, H. Hartmann, M. Reuther, C. Zobel, T. Lorenz, A. Tanaka, N. B. Brookes, H. H. Hsieh, H.-J. Lin, C. T. Chen, and L. H. Tjeng, Phys. Rev. Lett. 97, 176405 (2006).

[37] R. Eder, Phys. Rev. B 81, 035101 (2010).

[38] D. B. Chesnut, J. Chem. Phys. 40, 405 (1964).

[39] J. Wajnflasz, Phys. Status Solidi 40, 537 (1970).

[40] C. P. Slichter and H. G. Drickamer, J. Chem. Phys. 56, 2142 (1972).

[41] R. Zimmermann and E. König, J. Phys. Chem. Solids, 38, 779 (1977).

[42] B. C. Sales and D. K. Wohlleben, Phys. Rev. Lett. 35, 1240 (1975).

[43] W. Franz, F. Steglich, W. Zell, D. Wohlleben, and F. Pobell, Phys. Rev. Lett. 45, 64 (1980).

[44] M. Croft, J. A. Hodges, E. Kemly, A. Krishnan, V. Murgai and L. C. Gupta, Phys. Rev. Lett. 48, 826 (1982).

[45] E. V. Sampathkumaran, L. C. Gupta, R. Vijayaraghavan, K. V. Gopalakrishnan, R. G. Pillay and H. G. Devare, J. Phys. C: Solid State Phys. 14, L237 (1981).

[46] I. Felner and I. Nowik, Phys. Rev. B 33, 617 (1986).

[47] A. Mitsuda, H. Wada, M. Shiga, H. Aruga Katori and T. Goto, Phys. Rev. B 55, 12474

(1997) ; H. Wada, A. Nakamura, A. Mitsuda, M. Shiga, T. Tanaka, H. Mitamura and T. Goto, J. Phys.: Condens. Matter 9, 7913 (1997).

[48] The small difference in size between $\mathrm{Pr}^{3+}$ and $\mathrm{Sm}^{3+}$ allows a fine tuning the VSST.

[49] G. Y. Wang, T. Wu, X. G. Luo, W. Wang, and X. H. Chen, Phys. Rev. B 73, 052404 (2006);

G. Y. Wang, X. H. Chen, T. Wu, G. Wu, X. G. Luo, and C. H. Wang, Phys. Rev. B 74, 165113 (2006).

[50] R. Fehrenbacher and T. M. Rice, Phys. Rev. Lett. 70, 3471 (1993).

[51] S. W. Biernacki, Phys. Rev. B 74, 184420 (2006).

[52] M. Rotter, Z.-S. Wang, A. T. Boothroyd, D. Prabhakaran, A. Tanaka, and M. Doerr, Sci. Rep. 4, 7003 (2014).

[53] R. H. Mitchell, Perovskites: Modern and Ancient, Almaz Press, Ontario, Canada (2002).

[54] K. Asai, O. Yokokura, N. Nishimori, H. Chou, J. M. Tranquada, G. Shirane, S. Higuchi, Y. Okajima, and K. Kohn, Phys. Rev. B 50, 3025 (1994).

[55] J.-Q. Yan, J.-S. Zhou, and J. B. Goodenough, Phys. Rev. B 69, 134409 (2004).

[56] K. Knížek, Z. Jirák, J. Hejtmánek, M. Veverka, M. Maryško, G. Maris, and T. T. M. Palstra, Eur. Phys. J. B. 47, 213 (2005). 
[57] P. M. Raccah and J. B. Goodenough, Phys. Rev. 155, 932 (1967).

[58] G. Demazeau, M. Pouchard, Z. Li-Ming, and P. Hagenmuller, Z. Anorg. Allg. Chem. 555, 64 (1987).

[59] We use the notation in square brackets to refer to an amount per formula unit.

[60] Z. Ropka and R. J. Radwanski, Phys. Rev. B 67, 172401 (2003).

[61] For a review, see for instance, P. Gütlich and H. A. Goodwin, Top. Curr. Chem. 233-235, 1 (2004).

[62] Z. Jirák, J. Hejtmánek, K. Knížek, M. Maryško, P. Novák, E. Šantavá, T. Naito, and H. Fujishiro, J. Phys.: Condens. Matter 25, 216006 (2013).

[63] We used for $\mathrm{Pr}^{4+}$ the energy levels calculated for the isoelectronic $\mathrm{Ce}^{3+}$ ion.

[64] E. V. Sampathkumaran, K. H. Prank, G. Kalkowski, G. Kaindl, M. Domke, and G. Wortmann, Phys. Rev. B 29, 5702 (1984).

[65] See Supplemental Material at http :xxx

[66] J. Baier, S. Jodlauk, M. Kriener, A. Reichl, C. Zobel, H. Kierspel, A. Freimuth, and T. Lorenz, Phys. Rev. B 71, 014443 (2005).

[67] T. Kyomen, Y. Asaka, and M. Itoh, Phys. Rev. B 71, 024418 (2005). 